\title{
Adaptation en microtest de la technique de séroneutralisation par la méthode cinétique pour la recherche et le titrage des anticorps neutralisant le virus de la peste bovine
}

\author{
par M. RIOCHE
}

\begin{abstract}
RÉSUMÉ
L'auteur décrit une adaptation en microtest de la technique de séroneutralisation du virus de la peste bovine par la méthode cınétique mise au point par BOURDIN et BERNARD (1967). L'analyse statistique des résultats obtenus sur 453 sérums de ruminants testés qualitativement à la fois par la méthode classique et par le microtest ne permet pas de mettre en évidence une différence entre les deux méthodes qui sont donc superposables et peuvent être utilisées indifféremment dans la recherche qualitatıve des anticorps. La microméthode a l'avantage d'éconamiser temps ef réactifs. L'étude statistique des résultats obtenus dans le titrage des anticorps de 116 sérums montre une différence très signıficative entre les deux méthodes mais aussi qu'il existe une liaison très forfe entre elles. Lorsque le titrage des anticorps porte sur de grands effectifs, le microtest peut aussi être utllsé car les avantages qu'il présente compensent son manque éventuel de sensibilité par rapport à la méthode classique.
\end{abstract}

Les techniques immunologiques tendent actuellement à la miniaturisation en raison des avantages que les microméthodes offrent en sérologie : rapidité, faible encombrement, économie de réactifs et facilité de lecture. Connues depuis plusieurs années, (BARSKY ef LEPINE, 1954) (TAKATSY, 1955), ces techniques sont actuellement utilisées en pratique courante dans diverses réactions sérologiques telles que l'hémagglutination et l'inhibition de l'hémagglutination (SEVER, 1962), la fixation du complément (SEVER, 1962) (CASEY, 1965) ou la réaction de neutralisation (SALIM; 1967).

L'adaptation en micratest de la méthode cinétique de séro-neutralisation décrite en 1967 par BOURDIN et BERNARD pour la recherche des anticorps neutralisant le virus de la peste bovine, se justifie par le grand nombre d'examens sérologiques actuellement effectués chez les ruminants de l'Afrique de l'Ouest pour l'appréciatıon des résultats des campagnes de vaccination contre la peste bovine ef aussi pour les recherches épidémiologiques chez les petits ruminants.

\section{I. - MATÉRIEL ET MÉTHODE}

\section{1) Matériel.}

Nous $n$ 'indiquerons ici que le matériel destiné spécialement à la réalisation du microtest : 
a) Plaques en matière plastique transparente de $124 \times 81 \mathrm{~mm}$ contenant 96 cupules de $0.4 \mathrm{ml}$ d. fond plat (Limbro Chemical réf. 15 Fb 96). Ces plaques sont préalablement lavées, cupule par cupule dans une solution d'OMO (produit à base de Teepol) puis rincées 10 fois à l'eau courante, 3 fois à l'eau désionisée, 3 fois à l'eau tridistillée. Après séchage d̀ l'étuve, elles sont stérilisées dans l'alcool à $95^{\circ}$ pendant $30 \mathrm{mn}$ puis séchées dans une hotte à rayons uitraviolets pendant 3 à 4 heures. Elles sont conservées en boîte de Pétri stériles fermées hermétiquement, sı leur utilisation est différée.

b) Pipettes compte-gouttes en verre à chimie : ces pipettes, préparées en laboratoire, forment des gouttes de $0,015 \mathrm{ml} \pm 5 \mathrm{p}$. 100. Il est possible aussı d'utiliser des aiguilles de $6 / 10$ montées sur tige de verre $(1$ goutte $=0,015 \mathrm{ml})$.

\section{2) Méthode.}

La microtechnique décrite ici n'est qu'une adaptation de la méthode classique mise au point par BOURDIN et BERNARD en $1967\left(^{*}\right)$. Les substances entrant en récction sont les mêmes, la réalisation identıque. Seules les quantités de réactıfs changent mais leurs proportions relatives restent les mêmes afin de pouvoir comparer les résultats obtenus par les deux méthodes.

Ainsi dans la méthode classique, les auteurs mettent en contact $0,05 \mathrm{ml}$ de sérum à étudier décomplémenté avec $0,5 \mathrm{ml}$ d'une suspension de virus bovipestique représentant I DICT 100. Après 1 heure d'étuve à $37^{\circ} \mathrm{C}$, ils ajoutent $0,5 \mathrm{ml}$ d'une suspension cellulaire titrant 100.000 cellules $/ \mathrm{ml}$ de la lignée cellulaire permanente MDBKC (cellules épithélıales de rein de bovin adulte) établie en 1958 par MADIN ef DARBY. Le microtest, lui, requiert les quantités suivantes: $0,015 \mathrm{ml}$ de sérum décomplémenté à étudier (1 microgoutte), $0,15 \mathrm{ml}$ de virus titrant I DICT 100 dans $0,5 \mathrm{ml}$. La dilution finale de sérum est donc $10^{-1}$ comme dans la méthode classique et la quantité de cellules introduites après la neutralisation est proportionnellement équivalente: $0,15 \mathrm{ml}$ d'une suspension comptant 100.000 cellules/ml.

(*) Cette méthode a aussi été adaplée au titrage des anticorps (RIOCHE 1969). a) Réalisation de la technique qualitative : de même que dans lo technique classique 3 tubes sont utilisés pour chaque sérum, nous utilisons, par sérum, 3 cupules qui reçoivent :

1re cupule : TÉMOIN SÉRUM :

$0,15 \mathrm{ml}$ de milieu de culture,

$+0,015 \mathrm{ml}$ de sérum,

1 heure di $37 \mathrm{oC}$,

$+0,15 \mathrm{ml}$ de suspension cellulare.

2 et $3^{\text {es }}$ cupules : RÉACTION :

$0,015 \mathrm{ml}$ de sérum,

$+0,15 \mathrm{ml}$ de suspension de virus

1 heure à $37^{\circ} \mathrm{C}$,

$+0,15 \mathrm{ml}$ de suspension cellulaire.

Ensuite les plaques sont recouvertes d'un plastique adhésif et remises à $37^{\circ} \mathrm{C}$ pendant 5 jours.

b) Réalisation de la technique quantitative : elle est identique à la précédente à la différence que I'on utılise pour chaque sérum à tester :

- 2 cupules «témoin sérum 》

- 2 cupules « réaction» recevant une microgoutte de sérum non dilué, 2 autres cupules recevant 1 microgoutte de sérum dilué au $1 / 2$, ainsi de suite jusqu'aux dilutions terminales de 1/320 pour les sérums de bovins (1 microgoutte de sérum dilué au 1/32) et $1 / 80$ pour les sérums de petits ruminants $(1$ microgoutte de sérum dilué au 1/8).

A partir de la dilution au $1 / 20$, le sérum entrant en réaction est considéré comme insuffisant pour la nutrition des cellules et la suspension cellulaire est alors additionnée de 4 p. 100 de sérum normal de bœuf décomplémenté.

Le titre neutralisant du sérum est donné par la plus haute dilution dans laquelle le tapis cellulaire est soit indemne, soit partiellement détruit par le virus dans les deux cupules. SI cette dilution est $I / N$ nous admettons que le sérum considéré titre «N» unités neutralisant | DICT 100 de virus bovipestıque $/ \mathrm{ml}$.

Quelle que soit la méthode utilisée, nous ajoutons 5 «témains cellules» et 5 «témoins virus 》. Les cupules «témoıns cellules» reçoivent $0,015 \mathrm{ml}$ de sérum décomplémenté (de bœuf, de mouton ou de chèvre selon l'espèce animale faisant l'objet de la séro-neutralisation) et $0.15 \mathrm{ml}$ de milieu de culture. Après une heure 
TQBZIFAL $\because a$

\begin{tabular}{|c|c|c|c|c|}
\hline \multirow{2}{*}{$\begin{array}{c}\text { Espece et provenance } \\
\text { des sérums }\end{array}$} & \multicolumn{2}{|c|}{ Résultats positif́s } & \multicolumn{2}{|c|}{ Résultats négatifs } \\
\hline & Microtest & $\begin{array}{l}\text { Méthode } \\
\text { classique }\end{array}$ & Microtest & $\begin{array}{l}\text { Méthode } \\
\text { classique }\end{array}$ \\
\hline Bovms. Région de Saint-Louis & 129 & 126 & 17 & 20 \\
\hline Bovins. Sierra-Lecne & 40 & 43 & 13 & 10 \\
\hline Bovins. Région de kedougrou & 44 & 44 & 19 & 19 \\
\hline Petits ruminants. Casamance & $5 I$ & 47 & 43 & 47 \\
\hline Petits ruminants, Dahomey & 40 & 39 & 57 & 58 \\
\hline Total. & 304 & 299 & 149 & 154 \\
\hline
\end{tabular}

à $37^{\circ} \mathrm{C}$, on ajoute $0,15 \mathrm{ml}$ de suspension cellulaire. Après 5 jours à $37^{\circ} \mathrm{C}$, les tapis cellulaires doivent présenter un aspect normal et être indemnes de lésions.

Les cupules «témoins virus » reçoivent $0,015 \mathrm{ml}$ de sérum décomplémenté dépourvu d'antıcorps, $0,15 \mathrm{ml}$ de suspension de virus (I DICT 100 dans $0,5 \mathrm{ml}$ ) puis après une heure à $37^{\circ} \mathrm{C}, 0,15 \mathrm{ml}$ de suspension cellulaire. Après 5 jours à $37^{\circ} \mathrm{C}$ les tapis cellulaires doivent être totalement détruits par le virus.

\section{II. - RÉSULTATS OBTENUS}

Afin de savoir si la microméthode donne des résulitats comparables à ceux de la méthode classique en tubes à hémolyse, tous les essais ont été effectués par les 2 méthodes. Pour réaliser des conditions expérimentales identiques, lors de chaque essai, la même suspension de virus et la même suspension de cellules ont servi à la réalisation des deux méthodes. Les réponses sérologiques fournies par les deux méthodes sont les suivantes :

a) Technique qualitative :

Cette technique utılısant deux tests par échontillon de sérum, trois résultats différents peuvent être observés :

1) Les deux tapis cellulaires sont indemnes (le sérum a neutralisé le virus) : résultat positif.

2) Un tapis sur deux est indemne : résultat douteux.

3) Les deux tapis cellulaıres sont détruits par le virus (le sérum ne contient pas d'anticorps) : résultat négatif.
Les ruminants dont le sérum dilué au 1/10 donne un résultat douteux étant considérés comme non immuns, nous considérerons ces résultats comme négatifs.

Dans ces conditions les séro-neutralısations effectuées sur 191 sérums de petits ruminants et 262 sérums de bovins par les deux techniques qualitatives donnent les résultats figurant dans le tableau I.

b) Technique quantitative :

116 sérums de bovins ont été testés. Les titres obtenus par chacune des deux méthodes sont consıgnés dans le tableau II et représentés graphiquement dans les histogrammes I ef II.

\section{TABLEAU NI ${ }^{\circ} I I$}

\begin{tabular}{|c|c|c|}
\hline \multirow{2}{*}{$\begin{array}{c}\text { Titre des sérums } \\
\text { en unités }\end{array}$} & \multicolumn{2}{|c|}{$\begin{array}{c}\text { Nombre de sérums } \\
\text { ayant ce titre }\end{array}$} \\
\cline { 2 - 3 } reutralisantes & $\begin{array}{c}\text { par le } \\
\text { microtest }\end{array}$ & $\begin{array}{c}\text { par la } \\
\text { méthade } \\
\text { classique }\end{array}$ \\
\hline 10 & 18 & 13 \\
20 & 28 & 25 \\
40 & 39 & 34 \\
80 & 21 & 23 \\
160 & 9 & 20 \\
320 & 1 & 1 \\
\hline Total & 116 & 116 \\
\hline
\end{tabular}

\section{III. - ANALYSE STATISTIQUE DES RÉSULTATS}

La comparaison des deux méthodes en technique qualitative faisant intervenir un caractère qualitatif, c'est par le test du $\chi^{2}$ de PEARSON que les résultats pourront être analysés. 
Fistogramme I

Distribution des sérums en Eanction de leur titre déterminé par le microtest
Histogramme II

Distribution des sérums en fonction de leur titre déterminé par la méthode classique

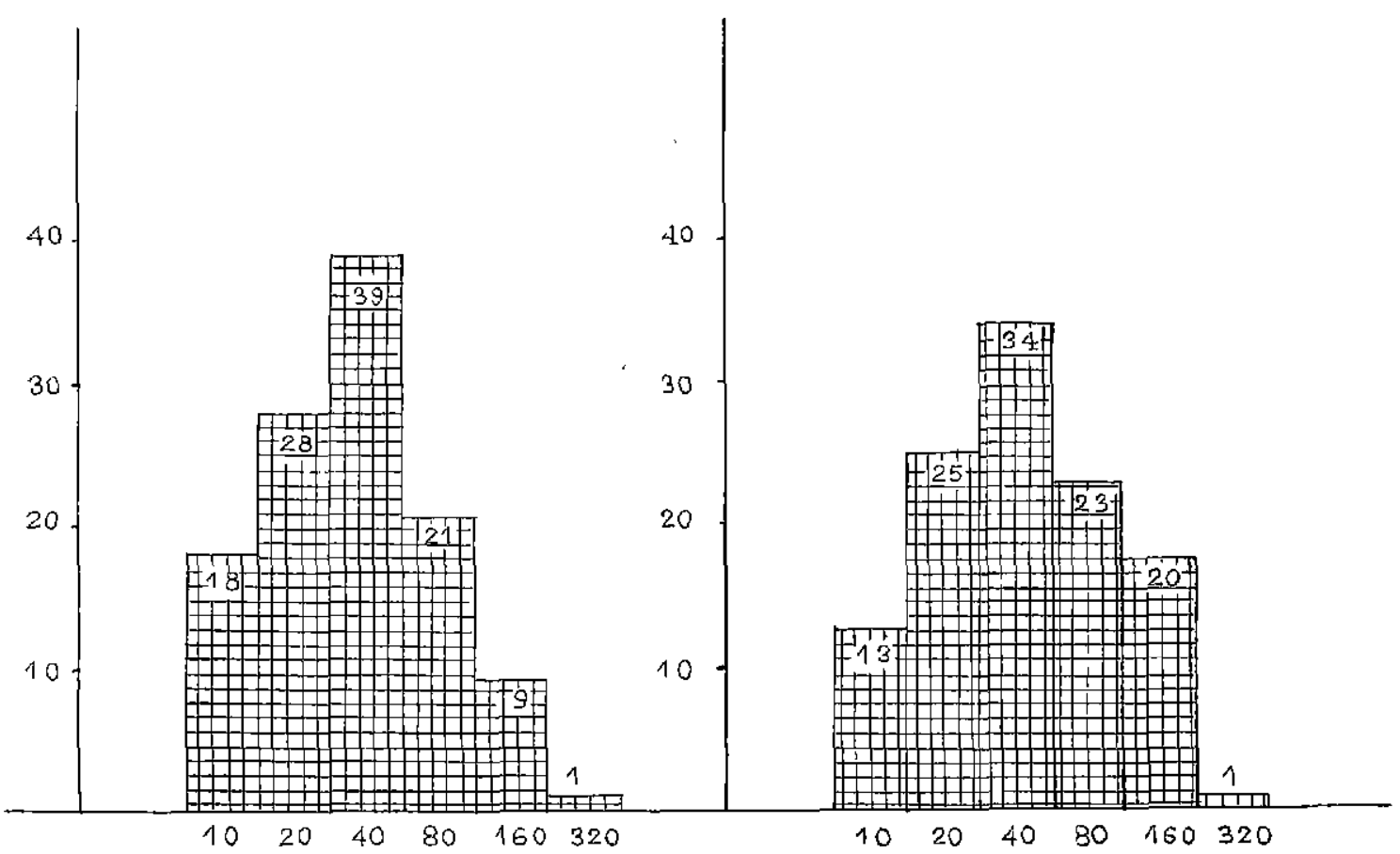

En abcisses: titre des sérums

En ordonnées : nombre de sérums donnant ces titres

Au contraire les techniques de titrages portent sur un caractère quantitatif et c'est le test de l'écart rédult qui permettra de comparer les deux méthodes.

a) Analyse des résultats obtenus par les techniques qualitatives $\left(^{*}\right)$ :

Le test $d u \chi^{2}$ comparant des répartitions observées à des répartitions théoriques, celles-ci peuvent être calculées à partir des résultałs consignés dans le tableau no 1 et l'on calcule les effectifs théoriques suivants :

(*) Dans la suite de l'exposé toutes les valeurs portant l'indice $\langle\mu »$ concernent la microméthode alors que l'indice « $\mathrm{c} »$ concerne les valeurs calculées pour la méthode classique.

$$
\begin{aligned}
- \text { Positifs }(+) 《 c » & =301,5 \\
- \text { Négatifs }(-)\langle c » & =151,5 \\
\text { et }+\langle\mu » & =301,5 \\
-\langle\mu » & =151,5
\end{aligned}
$$

On obtient alors une valeur de $\chi^{2}=0,124$ pour un degré de liberté, correspondant à un risque $\alpha$ compris entre 0,90 et 0,50 . Cette valeur est très inférieure d̀ 3,841 qui correspond au $\chi^{2}$ pour un risque $\alpha=0,05$. II n'existe donc pas de différence significative entre les résultats obtenus par l'une ef l'autre méthode.

b) Analyse des résultats obtenus par les méthodes quantitatives :

Chaque sérum ayant été examiné par les deux méthodes, le procédé le plus correct pour 
effectuer la comparaison est la méthode des séries appariées. L'écart réduit est :

$\varepsilon=\frac{m}{s \sqrt{n}}$ où $m$ est la moyenne des différences des titres pour chaque couple de mesure. Pour 116 sérums nous obtenons alors :

$$
\mathrm{m}=11,29 \text { et } \mathrm{S}^{2}=1562,6 \text { d'où } \varepsilon=3,077
$$

Cette valeur est nettement supérieure à la valeur 1,96 qui correspond à un risque de 0,05. Elle correspond d̀ une valeur de $\alpha$ comprise entre 0,01 et 0,001 . Ce résultat permet de conclure que les différences constatées dans le titrage des anticorps par les deux méthodes sont significatives.

Puisque le titre neutralisant d'un sérum varie en fonction de la méthode utilisée, il est utile de compléter l'étude statistique des résultats observés en cherchant si les deux méthodes de titrage sont cependant liées.

La recherche de l'existence d'une liaison se falt par le calcul du coefficient de corrélation, qui peut s'effectuer à partir du tableau ill dans lequel nous avons procédé à un changement d'unité. C'est cinsi que la valeur $x$ du titre est remplacée par $x^{\prime}=\log 2 \frac{x}{10}$. Aux valeurs de $x^{\prime}=0,1,2,3,4$ et 5 correspondent les valeurs d'origine $x=10,20,40 \ldots 320$.

TABLEAU $I^{\circ}$ III

\begin{tabular}{|c|c|c|c|c|c|c|}
\hline & 0 & 1 & 2 & 3 & 4 & 5 \\
\hline 0 & 6 & 4 & 3 & - & - & - \\
\hline 1 & 5 & 11 & 9 & - & - & - \\
\hline 2 & 3 & 10 & 18 & 3 & - & - \\
\hline 3 & 2 & 3 & 6 & 11 & 1 & - \\
\hline 4 & - & - & 3 & 7 & 9 & 1 \\
\hline 5 & - & - & - & - & 1 & - \\
\hline
\end{tabular}

Les calculs permettent d'obtenir un coefficient de corrélation :

$$
\text { ir }=0,7
$$

et une valeur $t=10,4$ pour $114 \mathrm{~d}$. d. I.

Cette valeur élevée de $t$ indıque que les deux techniques de tıtrage sont très fortement liées, le risque correspondant étant de loin inférieur à 0,05 .

\section{IV. - CONCLUSIONS}

L'analyse statistique des résultats observés ne nous a pas permis de mettre en évidence une différence entre les deux technıques de séroneutralisation cinétıque qualıtative. Ces deux méthodes sont donc comparables et Il semble qu'elles puissent être utılisées indifféremment pour la détection des anticorps neutralisant le virus de la Peste Bovine chez les ruminants. La microméthode présente l'avantage de sa rapidité d'exécution et de sa facilité de lecture.

Au contraire et bien que les deux techniques soient sıgnificatıvement lıées, microméthode et méthode classique ne donnent pas des réponses identiques pour le titrage des anticorps puisque l'analyse statisłıque des résultats révèle une différence très significative. Le titre moyen en anticorps $(49,4)$ obtenu par le microtest est inférleur au titre moyen $(63,3)$ observé dans la méthode classique. La microtechnique fournit donc des titres en général moins élevés que la méthode classıque. Ce manque de sensibilité des microméthodes est d'ailleurs reconnue d̀ l'heure actuelle.

En conséquence, nous estimons que dans la pratique, le microtest peut remplacer avantageusement la méthode classıque pour la détection des antıcorps neutralisantle virus de la Peste Bovine.

Il en sera de même pour le titrage des anticorps lorsque les recherches porteront sur de grands nombres (appréciation de l'état d'ımmunité naturelle ou acquise d'un grand effectif par exemple), l'économie de temps et de réactifs que permet de réaliser la microméthode compensant alors largement le manque de sensibilité qu'elle présente peut-être par rapport à la méthode classique.

\section{Remerclements.}

Nous adressons nas vifs remerciements au Docteur BARME, Chef de Service à I'Institut Pasieur de Dakar qui a bien voulu nous aider de ses conseils dans l'onalyse statistique de nos résultats.

Institut d'Elevoge et de Médecine vétérnaure des Pays tropicaux Masons-Alfort

Laboratore notional de l'Elevage et de Recherches vétérinares Dokor-Hónn 


\section{SUMMARY}

Adaptation in microfest of the seroneutralization fechnique by the kinetic method for the research and the titration of antibodies neutralizing the rinderpest virus

The author describes an adaptation in microtest of the seroneutralization technıque of the rinderpest virus by the kinetic method perfected by BOURDIN and BERNARD (1967). The statistical analysis of results obtaned on 453 serums of ruminants qualitatively tested at the same time by the classical method and by the microtest may not display a difference between the two methods. Then, they are superposable and may be employed indifferently in the qualitative research of antibodies. The micromethod economizes the time and the tests. The statistical study of results obtained in the antibodies titration of 116 serums indicates that a very significant difference is between the two methods but also a very imporant connection. When the antıbodies titration is applied to large numbers of sera, the microtest may be also utilized, because its advantages compensate its eventual deficiency of sensibility with regard to the classical method.

\section{RESUMEN}

Adaptación en microprueba de la tecnica de seroneutralización por el método cinetico para la búsqueda y el dosaje de los anticuerpos neutralizando el virus de la peste bovina

El autor describe una adaptación en microprueba de la tecnica de seroneutralización del virus de la peste bovina por el método cinetico mejorada por BOURDIN y BERNARD (1967). El analısıs estadístico de los resultatos obtenidos en 453 sueros de rumantes probados qualitativamente a la vez por el método clásico y por la microprueba no permite evidenciar una diferencia entre los dos métodos que pués son canjeables y que se puede utilizar indıferentemente para la búsqueda qualitatıva de los anticuerpos. El micrométodo tiene la ventaja de economizar tiempo y reactivos. El estudio estadístico de los resultados obtenidos en el dosaje de los antıcuerpos de 116 sueros muestra una diferencia muy significativa entre los dos métodos pero tambien que una relación muy importante existe entre ellos. Cuando se hace el dosaje de los anticuerpos en grandes efectivos, se puede tambien utilizar la microprueba por que las ventajas que tiene compensan su falta eventual de sensibilidad en relación con el método cläsico.

\section{BIBLIOGRAPHIE}

1. BARSKY (G.) et LEPINE (P.). - Microméthode de séro-neutralisation de la poliomyélite: emplai de cultures cellulaires sur plaques moulées en matière plastique. Ann. Inst. Pasteur, 1954, 86, 693-701.

2. BOURDIN (P.) et BERNARD (G.). - Application de la méthode de séro-neutralisation cinétique à la recherche des anticorps neutralisant le virus de la peste bovine chez les bovins, les ovins et les caprins. Rev. Elev. Méd. Vét. Pays trop., 1967, 20, 4, 531-536.

3. CASEY (L. H.), - Adaptation of Laboratory Branch complement fixation method to microtechnique, dans «Standardized diagnosis complement fixation method and adaptation to Microtest », U. S. Department of Health Education and Welfare, U. S. Government Printing Office, Washington, 1965. 
4. MADIN (S. D.) et DARBY (N. B.), - Established kidney cell lines of normal adult bovin and ovin origine. Proc. Soc. exp. Biol. and Med., 1958, 98, 574.

5. RIOCHE (M.). - Rapport annuel $1968 \mathrm{du}$ Laboraioire national d'Elevage ef de Recher* ches véférinaires, Dakar, 1969, 46 à 50.

6. SALIM (A. R.). - Neutralization of a Phlebotomus (Sandly) fever virus in Baby Hamster Kidney cells (BHK 21). Introduction of a simple microculture for plaque reduction tests. Trans. R. Soc, trop. Med. Hyg. 1967, 61, 2, 259-264.

7. SEVER (J. L.), - Application of a microtechnique to viral serological investigations. J. Immun., 1962, 88, 320-329.

8. TAKATSY (G.), - The use of spiral loops in serological and virological micromethods. Acta Microbiol. Acad. Sci. Hung., 1955, 3, $191-$ 203 (cité par Casey, L. H. et Sever J. L.). 